\title{
A New Area of the Mouse Anterior Hypothalamus Involved in Septohypothalamic Circuit
}

\author{
Noriko HORII-HAYASHI, Takayo SASAGAWA, and Mayumi NISHI* \\ Department of Anatomy and Cell Biology, Nara Medical University, Kashihara, Nara 634-8521, Japan
}

Received June 30, 2015; final version accepted July 23, 2015

\begin{abstract}
While the hypothalamus is now classified into more than ten compartments, uncharacterized areas remain. In this study, we show a new area in the anterior hypothalamus (AH) of mice, a triangular-shaped area between the paraventricular hypothalamic nucleus $(\mathrm{PVN})$ and the fornix, which is enriched in chondroitin sulfate proteoglycans (CSPGs). We designated this region perifornical area of the AH (PeFAH) based on its anatomical location. In Nissl staining, the PeFAH was distinguishable as an area of relatively low density. Immunohistochemical and DNA microarray analyses indicated that PeFAH contains sparsely distributed calretinin-positive neurons and densely clustered enkephalin-positive neurons. Furthermore, the PeFAH was shown to have bidirectional neural connections with the lateral septum (LS). We confirmed enkephalinergic projections from PeFAH neurons to the LS, and inversely, calbindin-positive LS neurons as afferents to the PeFAH. Finally, c-Fos expression analysis revealed that the activity of PeFAH neurons tended to be increased by psychological stressors but not by homeostatic stressors. These findings of neuronal subtypes and projections suggest that the region of the densely clustered encephalin-positive neurons in the PeFAH is comparable with the perifornical nucleus previously identified in rats.
\end{abstract}

KEYWORDS: anterior hypothalamus, lateral septum, enkephalin, psychological stress, perineuronal net

\section{Introduction}

While previous studies and brain atlases divide the hypothalamus into many nuclei and areas, uncharacterized regions remain. Recent studies suggest that hypothalamic neurons involved in the regulation of metabolism, stress response, and reproduction remain plastic through adulthood [1-6]. The paraventricular nucleus of the hypothalamus (PVN) is one such region that remains plastic throughout life and controls stress responses by the hypothalamicpituitary-adrenal axis. The number of synapses as well as the shape of glial cells in the PVN is dramatically altered in response to osmotic and psychological stressors [3,4].

Neural plasticity is modulated not only by changes in synapses but also by alterations in the structure of the extracellular matrix (ECM). Perineuronal nets (PNNs) are specialized ECM structures affecting neuronal plasticity, covering the somata and proximal dendrites of certain types of neurons with a lattice-like meshwork. PNNs are composed of molecules such as chondroitin sulfate proteoglycans (CSPGs), hyaluronan, hyaluronan and proteoglycan link proteins (HAPLNs), and some glycoproteins [7, 8]. Ocular dominance plasticity is restored by destruction of PNNs in the adult visual cortex following injection of chondroitinase $\mathrm{ABC}(\mathrm{ChABC})$ [9]. The same treatment in the adult amygdala causes fear memories easily erased [10].

Many studies have focused on PNNs in the cerebral cortex $(\mathrm{Cx})$ and limbic system, and the majority of PNNs in these regions surround parvalbumin (PARV)-positive inhibitory neurons [11-15]. However, few studies have exhibited formation and function of PNNs in the hypothalamus. The lectin Wisteria floribunda agglutinin (WFA) binds to carbohydrate structures terminated by $\mathrm{N}$-acetylgalactosamine (GalNAc) and is often used for detecting chondroitin sulfate and PNN [15-17]. When we observed PNN formation in the mouse hypothalamus using WFA staining, we found a strongly WFA-labeled region that is surrounded by the fornix, PVN, and the central division of the anterior hypothalamus (AHC). Since this region had not been identified either by the mouse brain atlas [18] or previous literature, we named this region the "perifornical area of the anterior hypothalamus (PeFAH)" based on its anatomical location and its lower neuronal density evaluated by Nissl staining (Fig. 1E). The aim of this review is a characterization of the PeFAH in terms of its neuronal components, neuronal connections, and neuronal reactivity. We first identified the neuronal subtypes in the PeFAH by histological and DNA microarray analyses. Second, we investigated the connections of this region by neuronal tract tracing. Third, to elucidate the reactivity of PeFAH neurons, we analyzed the pattern of c-Fos expression in the PeFAH as an indicator of activity in neurons after exposure to psychological or homeostatic stressors. 

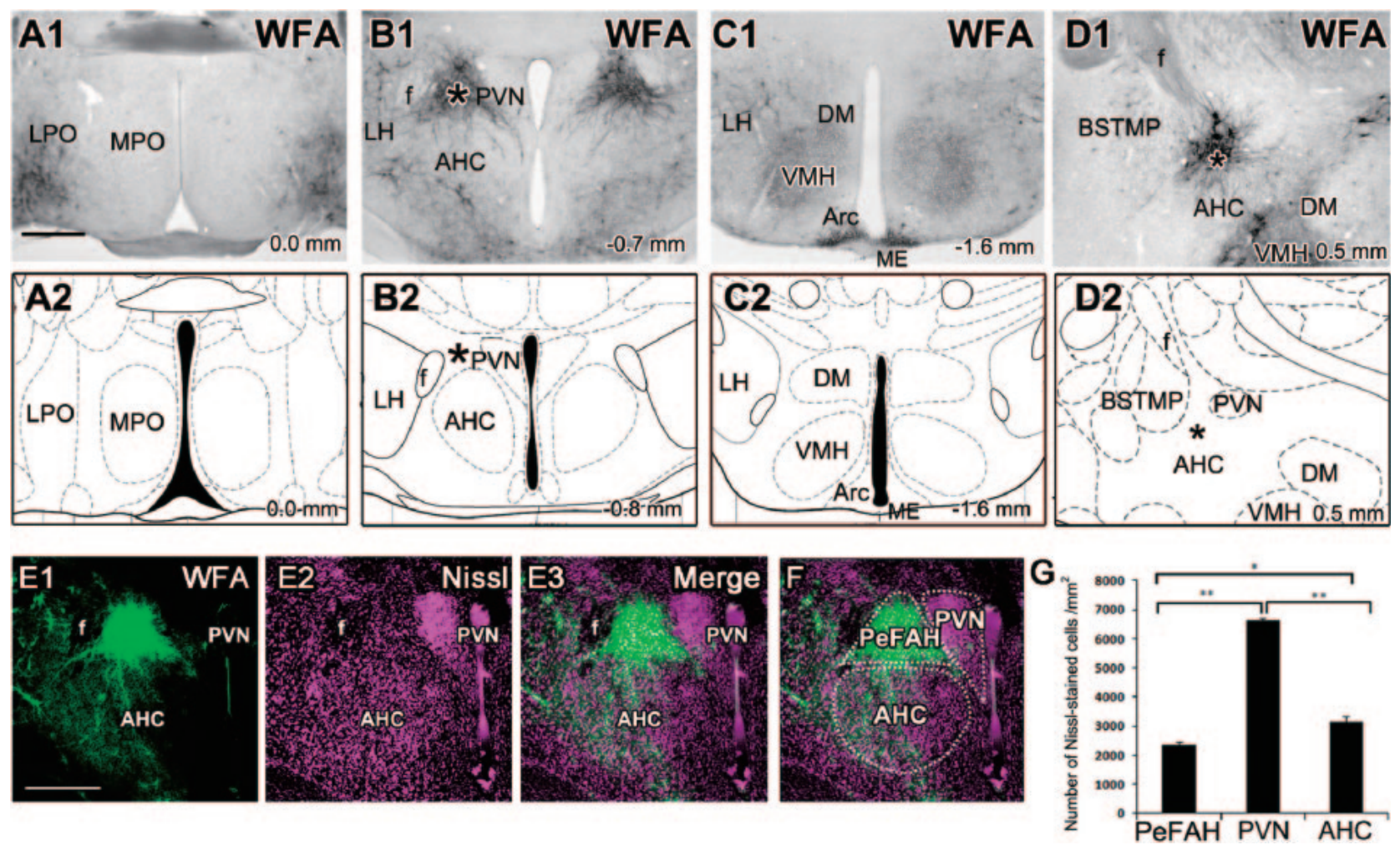

Fig. 1. WFA labeling reveals a new hypothalamic region between the PVN and the fornix

$\boldsymbol{A}-\boldsymbol{D}$, A series of WFA-labeled coronal (A1-C1) and sagittal (D1) sections and their approximate diagrams in the mouse brain atlas (A2-D2). Numerical values indicate the distance from bregma (A-C) or the midline (D). Note that the highly WFA-stained region in B1 or D1 (asterisks) is not designated in the coronal or sagittal atlas (B2, D2, asterisks). $\boldsymbol{E}, \boldsymbol{F}$, Double labeling of WFA and Nissl indicates that the WFA-labeled region can be identified as a triangle-shaped region with a sparse distribution of Nisslstained cells (E) and regional definitions of the PeFAH, PVN, and AHC (F). G, The graph shows the quantitative result for density of Nissl-stained cells in the PeFAH, PVN, and AHC, demonstrating a lower density in the PeFAH than that in the PVN and AHC (mean \pm SEM, one-way ANOVA, Tukey's post-hoc test, ${ }^{*} \mathrm{P}<0.01,{ }^{*} \mathrm{P}<0.0001, \mathrm{n}=3$ ). Arc: arcuate nucleus; AHC: central part of the anterior hypothalamic area; DM: dorsomedial hypothalamic nucleus; f: fornix; LH: lateral hypothalamic area; LPO: lateral preoptic area; ME: median eminence; MPO: medial preoptic nucleus; PeFAH: perifornical area of anterior hypothalamus; PVN: paraventricular nucleus; VMH: ventromedial hypothalamic nucleus. Scale bars $=500 \mu \mathrm{m}$ (A-D) (E, F).

\section{A new hypothalamic area enriched in CSPGs}

WFA labeling in the mouse hypothalamus showed that its signal was hardly observed in the medial preoptic nucleus (MPO) (Fig. 1A), PVN (Fig. 1B), and dorsomedial hypothalamic nucleus (DM) (Fig. 1C). In contrast, the signal was clearly detected in the lateral preoptic area (LPO) (Fig. 1A), lateral hypothalamic area (LH) (Fig. 1B, C), ventral side of the central part of the anterior hypothalamic area (AHC) (Fig. 1B), and VMH (Fig. 1C). In the arcuate nucleus (Arc), only the ventral border neighboring the median eminence (ME) was densely labeled with WFA (Fig. 1C). Notably, a region surrounded by the fornix, PVN, and AHC was densely labeled with WFA (Fig. 1B), which positioned at approximately $0.7 \mathrm{~mm}$ posterior from the bregma. This region that we designated the PeFAH was not specifically designated in the mouse brain atlas [18] (Fig. 1B2). We also checked the position of this region using sagittal sections, which indicated a strongly WFA-labeled region caudal to the medial posterior part of the bed nucleus of the stria terminalis (BSTMP) (Fig. 1D), which located at approximately $0.45 \mathrm{~mm}$ lateral from the midline. As in the coronal atlas, this WFA-labeled region is not specifically designated in the sagittal atlas (Fig. 1D2). Double staining of Nissl and WFA showed that the boundary of the WFA-labeled triangular region could be distinguished by Nissl staining (Fig. 1E). Nissl-stained cells in the PeFAH, PVN, and AHC, as shown in Fig. 1F, were quantitatively analyzed. The density of Nissl-stained cells in the PeFAH $\left(2356 \pm 107\right.$ cells $\left./ \mathrm{mm}^{2}, \mathrm{n}=3\right)$ was significantly lower than that in the PVN $\left(6644 \pm 50\right.$ cells $/ \mathrm{mm}^{2}, P<0.0001$, post-hoc $)$ and AHC $\left(3159 \pm 188\right.$ cells $/ \mathrm{mm}^{2}, P<0.01$, post-hoc $)$ (Fig. 1G). These results indicate that the PeFAH is a single compartmented area, distinguished from the PVN and AHC by WFA or Nissl staining.

WFA signal in the PeFAH disappeared at the site of a ChABC injection (Fig. 2A), indicating that this signal was specifically derived from the chondroitin sulfate (CS). Since a previous study demonstrated that WFA mainly recognizes a carbohydrate epitope on aggrecan, a major CSPG component of PNNs [19], we performed aggrecan 

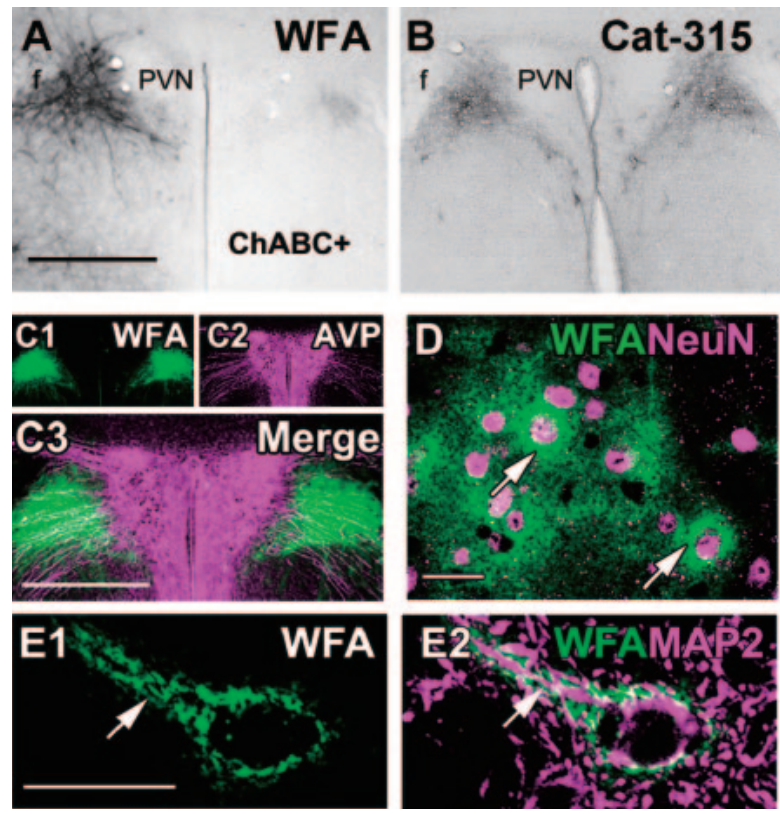

Fig. 2. PNN-positive neurons in the PeFAH

$\boldsymbol{A}$, The left side of the image shows a WFA-stained PeFAH between the fornix and PVN, while unilateral injection of chondroitinase $\mathrm{ABC}(\mathrm{ChABC})$ abolished WFA signal in the injected side (the right side of the picture). $\boldsymbol{B}$, Immunohistochemical staining with anti-Cat-315 antibody indicating an aggrecan-immunoreactive region with a triangular shape between the fornix and PVN. $\boldsymbol{C}$, Double labeling of WFA and AVP shows an adjacent but non-overlapping distribution of the PeFAH and PVN. $\boldsymbol{D}$, Double labeling of WFA (green) and NeuN (magenta) reveals NeuN-labeled neurons surrounded by PNN (arrows). E, Double labeling of WFA (green) and MAP2 (magenta) indicates the sheath of PNNs around a MAP2-labeled dendrite (arrows). f: fornix; PVN: paraventricular nucleus. Scale bars $=500(A, B)(C)$ and 50 (D) $(E) \mu \mathrm{m}$.

staining with anti-Cat-315 antibody. The Cat-315 immunoreactivity also exhibited the triangular shape of the region between the fornix and PVN (Fig. 2B). Double labeling of WFA and arginine vasopressin (AVP), a highly expressed neuropeptide in the PVN, revealed that WFA and AVP signals were adjacent but hardly overlapped (Fig. 2C). Additionally, double labeling of WFA and NeuN or microtubule associate protein 2 (MAP2) indicated that the WFA signal encompassed somata of NeuN-labeled neurons (Fig. 2D) or MAP2-labeled dendrites (Fig. 2E). These results indicate that the PeFAH includes PNN-positive neurons. However, WFA staining in the PeFAH is subtly incompatible with the common morphological structures of PNNs, showing a lattice-like meshwork [8]: WFA reactivity is particularly thick around somata but is diffuse around dendrites, and no clear lattice-like meshwork can be perceived. Thus, the ECM structures around PEFAH neurons seem not to be typical PNNs. Furthermore, unlike the LH and the $\mathrm{Cx}$, the expression of HPLN1, also known as cartilage link protein 1, which is an important molecular component of the highly specialized PNNs structures [20], was not detected PeFAH.

Interestingly, each hypothalamic nucleus/area, including the PeFAH, shows a characteristic staining pattern of WFA. For example, medial hypothalamic structures such as the medial preoptic area, the PVN, and the DM, show lower WFA stainability, while the lateral preoptic area and the LH include PNN-positive neurons. Thus, it is thought that each region has own characteristic extracellular environment, which may lead to differences in neural plasticity and/or cationic conditions. According to the hypothesis that PNNs limit plasticity, the plasticity of PeFAH neurons may be highly restricted by perineuronal and perisynaptic sheaths enriched in CSPGs. However, since animals lacking HAPLN1 are known to exhibit persistent visual plasticity [20], further studies are required to assess the functions of CSPGs in the PeFAH, which we plan to carry out.

\section{Characterization of the PeFAH by microarray and histological analyses}

Microarray analysis was performed to screen for neurotransmitters, peptides, and neuronal marker proteins expressed in the PeFAH and PVN. Gene expression levels are analyzed based on the log2-transformed signal intensity of each gene. Genes for glutamatergic (Slclal and Slcl7a6) and gamma-aminobutyric acid (GABA)-ergic (Gadl, Gad2, and Slc6al) markers showed high expression in both the PeFAH and PVN. However, monoaminergic (Slc6a2, Slc6a4, and Th) and cholinergic (Chat) markers showed lower expression in the PeFAH, while Th expression in the PVN was moderate. Compared to the PeFAH, the PVN showed higher expression levels for Avp, Crh, Oxt, and Sst, which encode for AVP, corticotropin-releasing hormone (CRH), oxytocin (OXT), and somatostatin, respectively, and lower expression levels for Penk, which encodes methionine-Enk (M-Enk) and leucine-Enk (L-Enk). Among genes for calcium-binding proteins, Calb2 was prominently expressed in both the regions. 

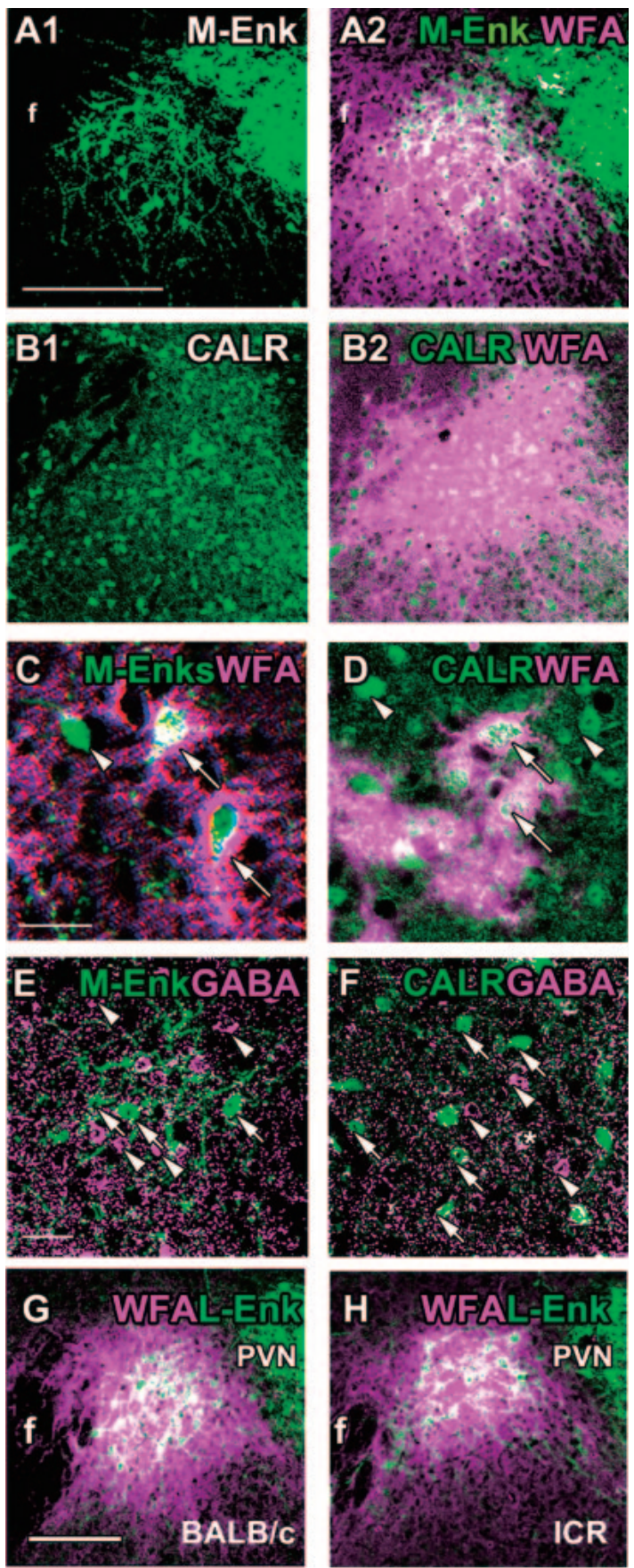

Fig. 3. Neuronal subtypes in the PeFAH

$\boldsymbol{A}-\boldsymbol{D}$, fluorescent images show double labeling of WFA (magenta) and M-Enk (green) or CALR (green) at lower (A, B) and higher (C, D) magnifications. M-Enk-positive neurons (A) were identified as a cluster in the dorsal location of the PeFAH, while CALR-positive neurons (B) were spread out in the whole of the PeFAH. Some M-Enk- (C) or CALR- (D) positive neurons are surrounded by PNNs (arrows), and some are not (arrowheads). $\boldsymbol{E}, \boldsymbol{F}$, Double labeling of GABA (magenta) and M-Enk (green) or CALR (green) showing that M-Enk- (E) or CALR- (F) positive neurons (arrows) are GABA-negative, and GABA-positive neurons (arrowheads) are M-Enk- or CALR-negative. $\boldsymbol{G}, \boldsymbol{H}$, WFA (magenta)/L-Enk (green) labeling in the colchicine-treated $\mathrm{BALB} / \mathrm{c}(\mathrm{G})$ or ICR $(\mathrm{H})$ mouse. A cluster of L-Enk positive neurons is observed in a WFA-labeled region between the PVN and the fornix. AHC: central part of the anterior hypothalamic area; PeFAH: perifornical area of anterior hypothalamus; PVN: paraventricular nucleus. Scale bars $=250(\mathrm{~A}, \mathrm{~B}, \mathrm{G}), 50(\mathrm{C}) \mu \mathrm{m}$.

Expressions of the majority of the above genes were investigated by immunohistochemistry, occasionally using colchicine-treated mice, in cases of detecting M-Enk, L-Enk, GABA, AVP, CRH, OXT, hypocretin, and vesicular glutamate transporter 2 (VGLUT2). Prominent somatic expressions of M-Enk, L-Enk, CALR (product of Calb2), and GABA were detected in the PeFAH (Fig. 3). However, substantial soma-like signals of the following neurotransmitters and calcium-binding proteins could not observed in the PeFAH: AVP, CRH, OXT, somatostatin, hypocretin (product of $H c r t$ ), PARV (product of Pvalb), calbindin (CALB, product of Calbl), choline acetyltransferase (product of Chat), and 
tyrosine hydroxylase (product of Th). Punctate signal of VGLUT2 (product of Slc17a6), probably derived from synaptic signal, was clearly detected in the PeFAH. Its soma-like signal was not detected even in colchicine-treated mice. Double labeling of WFA and M-Enk revealed a prominent cluster of Enk-positive neurons in a dorsal location in the PeFAH, in colchicine-treated mice (Fig. 3A). The same result was obtained when using anti-L-Enk antibody. Double labeling of WFA and CALR indicated that there were many CALR-positive neurons dispersed in the PeFAH (Fig. 3B). Higher magnification views of double labeling of WFA and M-Enk or CALR revealed that some of the Enkor CALR-positive neurons were surrounded by CSPGs (Fig. 3C, D). We also investigated GABA expression in Enkand CALR-positive neurons in colchicine-treated mice. Although some GABA-positive somata were detected in the PeFAH, both M-Enk- and CALR-positive neurons were GABA-negative (Fig. 3E, F). These results indicate that at least three types of neurons exist in the PeFAH: Enk-positive/GABA-negative, CALR-positive/GABA-negative, and GABA-positive. Furthermore, we analyzed different mouse strains. In both BALB/c and ICR strains, a strongly WFAlabeled region was observed between the fornix and the PVN, which contained a cluster of L-Enk-positive neurons (Fig. 3G, H).

The immunohistochemical results are consistent with those of DNA microarray analysis. Additional confirmation from in situ hybridization data from the Allen Brain Atlas, indicating that the genes Calb2 (Exp No. 79556662) and Penk (Exp. No. 74881286) are highly expressed in the region presumed to be the HDA. A few gadl-positive cells are detected in the same region, whereas the signals for Pvalb (Exp. No. 868) and Calbl (Exp. No. 79556672) are hardly detected.

\section{Neural networks of the PeFAH neurons}

Projections from the PeFAH were investigated with the anterograde tracer biotinylated dextran amine (BDA) (Fig. 4A). The micrograph of the injection site indicated specific injection of BDA into the PeFAH (Fig. 4B). A massive ipsilateral projection to the lateral septum (LS) was observed, in which BDA-reactive fibers were detected extensively (Fig. 4C). A few BDA-reactive fibers were also detected on the contralateral side. Projection of PeFAH neurons to the LS was confirmed by retrograde tracing with fluorogold (FG) (Fig. 4D), whose injection site is shown in Fig. 4E. Many FG-positive cells were observed on both sides of the PeFAH (Fig. 4F, G, H). Part of the region of FGpositive cells overlapped with preproenkephalin-positive and CALR-positive neurons (Fig. 4I, J), indicating that Enkand CALR-positive PeFAH neurons project to the LS.

Retrograde tracing with cholera toxin sbunit $\mathrm{B}(\mathrm{CTb})$ was then performed to identify afferents to the PeFAH (Fig. 5A), and the injection site was shown in Fig. 5B. CTb-positive cells were detected ipsilaterally over a wide extent of the LS (Fig. 5C). The majority of LS neurons are known to be GABAergic, and express CALB and CALR [21]. Lower-magnification views showed that CTb-labeled LS neuron distribution resembled CALB-positive neuron distribution (Fig. 5D). CTb-labeled neurons were partly overlapped with CALB-positive neurons, but not with CALRpositive neurons (Fig. 5E).

The medial hypothalamus and the LS are considered to have reciprocal neural connections, but the relevant neuronal subtypes and anatomical details have not yet been fully elucidated. Our tracing studies reveal direct inputs from CALBpositive LS neurons to the PeFAH; this finding is the first elucidation of LS neuron subtypes projecting to the hypothalamus. Since CALB-positive LS neurons are GABAergic [21], PeFAH neurons would presumably receive inhibitory regulation from LS neurons. Interestingly, a previous study in rats demonstrated that Enk-positive fibers from the hypothalamus terminate on CALB-positive neurons in the LS [22]. Therefore, several lines of evidence suggest the presence of a bidirectional connection between Enk-positive hypothalamic neurons and CALB-positive LS neurons.

\section{Neuronal responsiveness and possible functions}

To clarify the reactivity of PeFAH neurons, we investigated c-Fos expression after several forms of stimulation in the PeFAH, PVN, AHC, and LS. In group-housed and singly housed, non-stimulated controls, c-Fos expression was minimal in all regions analyzed. c-Fos expression in the PeFAH after the open field trial (a novelty-stress model) was significantly increased relative to that in the control group (Fig. 6A, C). In addition, significant increases were also observed in all other regions analyzed (Fig. 6A, C). Restraint stress, a severe type of stressor, also increased c-Fos expression in the PeFAH as well as all other regions (Fig. 6A, D). Similarly, aggression also increased c-Fos expression in the PeFAH and other regions (Fig. 6A, E). Neither dehydration nor subsequent rehydration affected c-Fos expression in the PeFAH and AHC, whereas dehydration remarkably increased c-Fos expression in the PVN and slightly but significantly increased it in the LS (Fig. 6F). After fasting and refeeding, there were no significant changes in c-Fos expression in the PeFAH and AHC, while c-Fos expression in the PVN after refeeding was significantly increased (Fig. 6G). Both fasting and refeeding slightly but significantly increased c-Fos expression in the LS (Fig. 6G). Double labeling of WFA and GR showed prominent GR expression in the PeFAH (Fig. 6B).

Many studies have revealed c-Fos expression patterns in the PVN in response to various forms of stimulation, whereas those of its neighboring regions are poorly understood. Increased c-Fos expression in the PeFAH was observed after restraint stress, the open-field test, and aggression, while the homeostatic stimuli dehydration/rehydration and fasting/refeeding did not change c-Fos expression in the PeFAH. Our assurance in the PeFAH results is verified by the 


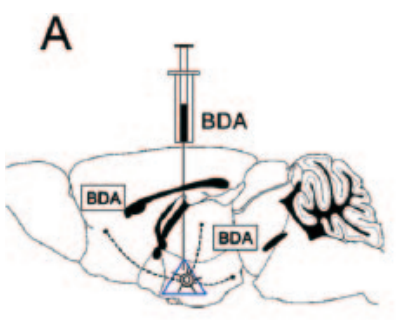

B
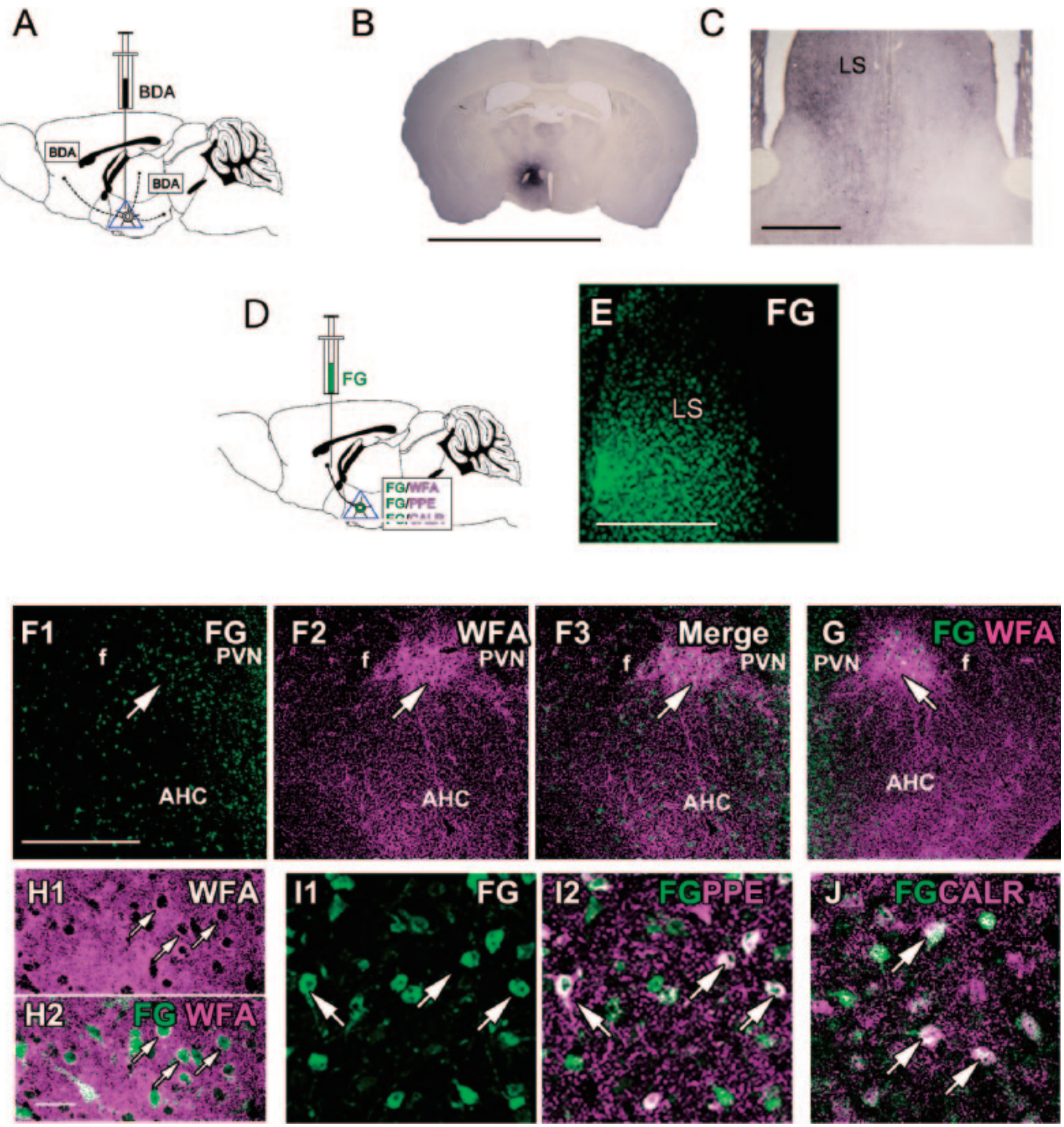

Fig. 4. Projections of DA neurons

$\boldsymbol{A}$, A schematic diagram illustrating anterograde tracing with BDA in the PeFAH. B, BDA reaction at the injection site (arrow). $\boldsymbol{C}$, Many BDA-reactive fibers are ipsilaterally observed in the LS. $\boldsymbol{D}$, A schematic diagram illustrating retrograde tracing with FG applied to LS neurons, which is followed by the detection of GF/WFA, FG/PPE, and GF/CALR in the PeFAH. E, FG signal in the injection site. $\boldsymbol{F}-\boldsymbol{H}$, Double labeling of FG (green) and WFA (magenta) shows FG-labeled neurons in the PeFAH in both ipsilateral (F, arrows) and contralateral (G, arrow) sides. Higher magnifications reveal FG-labeled neurons surrounded by PNNs (H, arrows). I, $\boldsymbol{J}$, Double labeling of FG (green) and PPE (magenta) or CALR (magenta) demonstrating overlap of FG-labeled neurons with PPE-positive (I, arrows) or CALR-positive (J, arrows) neurons. AHC: central part of the anterior hypothalamic area; LS: lateral septum. Scale bars $=5 \mathrm{~mm}(\mathrm{~B}), 500(\mathrm{C})(\mathrm{F}, \mathrm{G}), 250(\mathrm{E})$, and $50(\mathrm{H}) \mu \mathrm{m}$.

agreement of our PVN c-Fos results with published studies, showing enhancement by restraint, the open-field test, aggression, dehydration, and refeeding after fasting [23-25]. The manner of c-Fos expression in the PeFAH suggests that PeFAH neurons tend to respond to psychological stressors rather than to homeostatic stressors. Although we cannot exclude the possibility that PeFAH neurons have functional roles in homeostatic regulation, our findings of neural connections between PeFAH neurons and LS neurons also support this view, because the LS is known to play a crucial role in regulating emotion [26]. A recent optogenetic study reveals that the pathway from LS neurons to the AH regulates the level of stress-induced anxiety [27]. According to these data, the LS fibers labeled for optogenetic stimulation innervated in and around the presumed PeFAH and the AHC. Thus, PeFAH neurons may be involved in emotional regulation under stressful conditions. However, functional roles for Enk-positive neurons in the AH have not been elucidated. The present data will enable future optogenetic and chemogenetic studies to clarify the functional roles of the neural circuits between the hypothalamus and the LS.

\section{Summary}

In neuroanatomy, a "nucleus" is a cytoarchitectonic structure composed of a relatively compact cluster of neurons, 

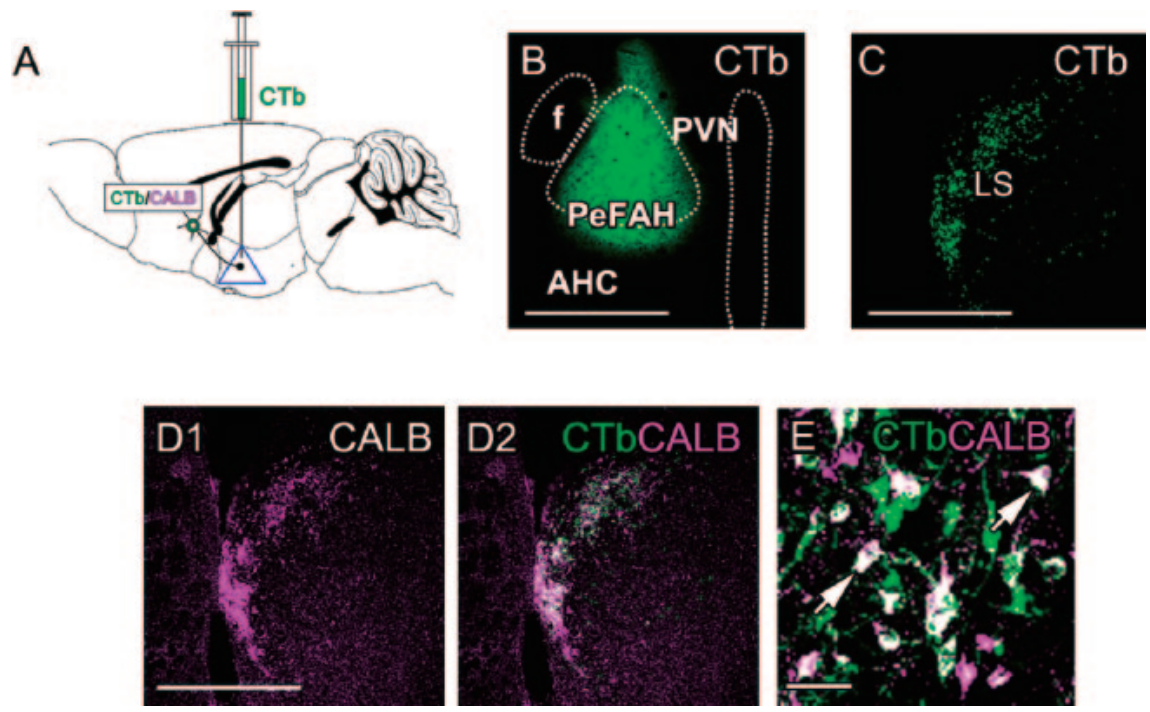

Fig. 5. Afferents of the PeFAH

$\boldsymbol{A}$, A schematic diagram illustrating retrograde tracing with $\mathrm{CTb}$ in the PeFAH, which is followed by immunohistochemical detection of CALB in the LS. B, A picture of the injection site. $\boldsymbol{C}$, CTb distribution in the LS. $\boldsymbol{D}, \boldsymbol{E}$, Dual images of CTb (green) and CALB immunoreactivity (magenta) in the LS showing similar distributions of these two signals at lower magnification (D) and overlapping of CTb-labeled neurons with CALB-positive neurons at single-cell level (E, arrows). Scale bars $=500$ (B,

$\mathrm{C}, \mathrm{D})$ and $50(\mathrm{E}) \mu \mathrm{m}$.

A

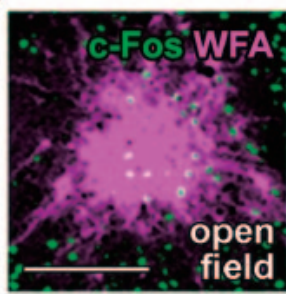

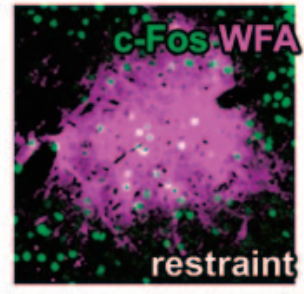

B

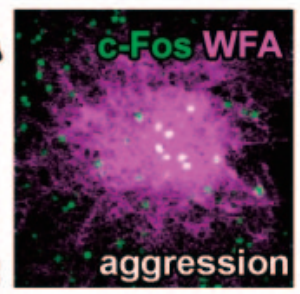

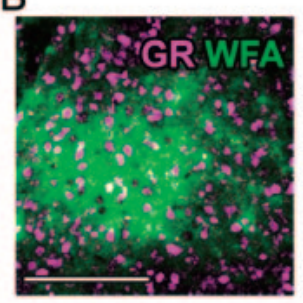
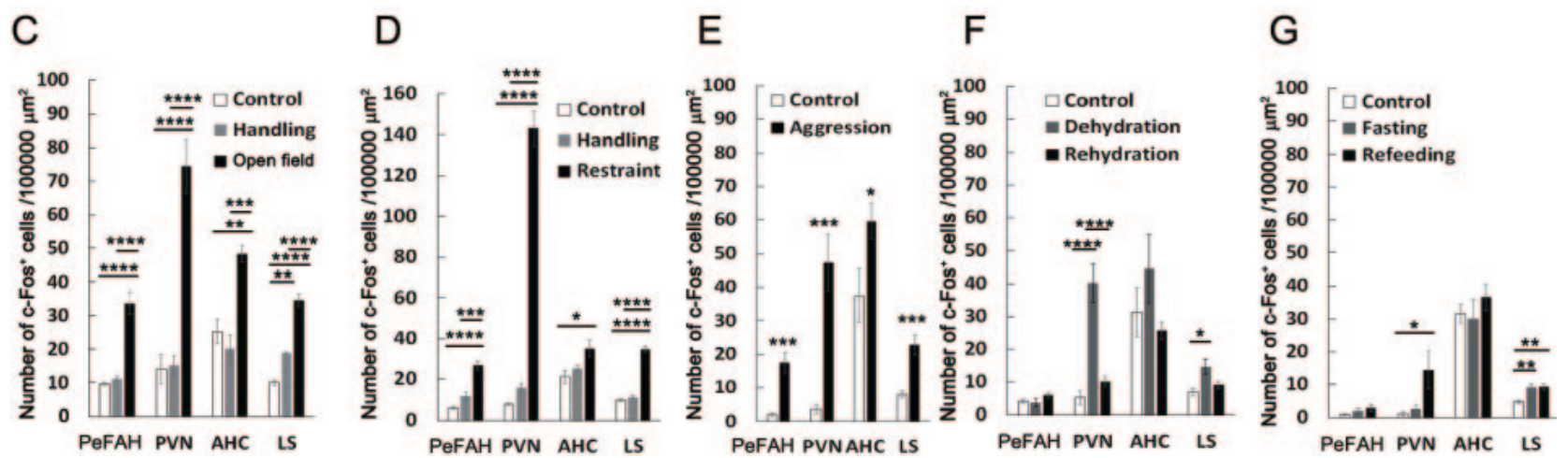

Fig. 6. c-Fos expression after the various kinds of stimuli

$\boldsymbol{A}$, Representative fluorescent images of c-Fos (green)/WFA (magenta) staining in the PeFAH area after the open field challenge (left), restraint stress (center), and aggression (right) groups. B, Representative fluorescent images of GR (magenta)/WFA (green) in the PeFAH. $\boldsymbol{C}-\boldsymbol{F}$, Graphs show the density of c-Fos-positive cells in open field (C), restraint (D), aggression (E), dehydration/rehydration $(\mathrm{F})$, and fasting/refeeding $(\mathrm{G})$. Data are mean \pm SE. Statistical differences were determined by oneway ANOVA with Tukey's post-hoc test $(\mathrm{C}, \mathrm{D}, \mathrm{F}, \mathrm{G})$ or t-test $(\mathrm{E}) .{ }^{*}, \mathrm{p}<0.05 ;{ }^{* *}, \mathrm{p}<0.01 ;{ }^{* * *}, \mathrm{p}<0.001 ;{ }^{* * * *}, \mathrm{p}<0.0001$. PeFAH: perifornical area of anterior hypothalamus; PVN: paraventricular nucleus; AHC: central part of the anterior hypothalamus; LS: lateral septum. Scale bars $=500(\mathrm{~A}, \mathrm{~B}) \mu \mathrm{m}$.

while "area" is used to refer to a region composed of sparsely distributed neurons. According to this definition, the PeFAH is considered an "area." A large number of studies using mice as well as rats report the neuroanatomy of the PVN, while few studies address that of its neighboring regions specifically in mice. In rats, the peri-PVN is an already characterized region located peripheral to the PVN [28]. However, whereas the histological location of the peri-PVN is very similar to that of the PeFAH, its neuronal components as well as its projection targets are very distinct from those 


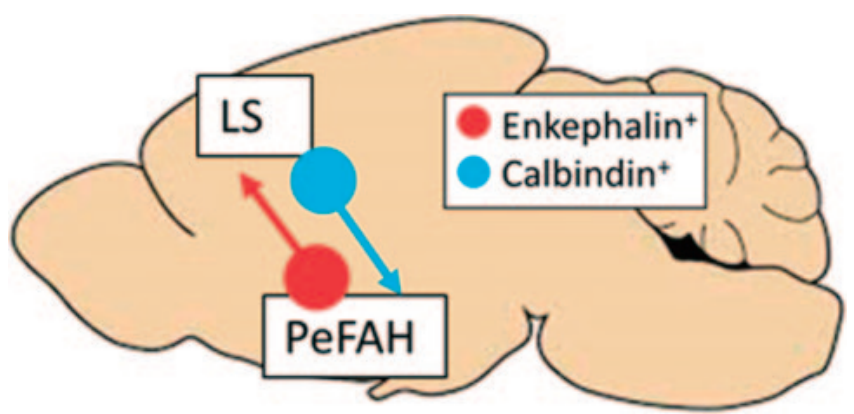

Fig. 7. Schema of the neural networks of the PeFAH

A schema shows a bidirectional connection between Enk-positive PeFAH neurons and CALB-positive LS neurons.

of the PeFAH, the peri-PVN in rat being composed of abundant GABAergic neurons projecting to the PVN [28]. Previous studies in guinea pig report that a cluster of Enk-positive neurons projecting to the LS is located between the fornix and the PVN. This cluster is named the magnocellular dorsal nucleus (MDN) [29,30]. Subsequently, a region homologous with the guinea pig MDN, the perifornical nucleus (PeF), was identified in rats, and is located around the fornix at PVN levels. Considering neuron subtypes and projections, the cluster of Enk-positive neurons within the PeFAH would be comparable with the guinea pig MDN and the rat PeF. However, the whole of the PeFAH is larger than its Enk-positive region and is further characterized by a lower neuronal density and the presence of sparse CALRpositive neurons. Thus, it is reasonable to call the PeFAH a perifornical area [31].

In conclusion, we found and characterized a previously unidentified region in the anterior hypothalamus in the mouse brain. We designated this area, PeFAH, which locates between the fornix and the PVN. In addition to its WFA staining, the PeFAH is discriminable as an area of sparse Nissl staining and as a triangular area by immunohistochemical staining with anti-Cat315 antibody. Moreover, histological and DNA microarray analyses elucidated that the PeFAH contains a cluster of Enk-positive neurons and CALR-positive neurons, both of which are GABA-negative. Neuronal tract tracing analyses clarified that the major projection target of PeFAH neurons is the LS and that they receive input from calbindin-positive LS neurons, showing a bidirectional neural connection between the PeFAH and LS (Fig. 7). Finally, c-Fos experiment suggests that PeFAH neurons have reactivity to psychological stressors, whereas exhibiting no or low reactivity to homeostatic stressors.

\section{Acknowledgements}

We thank Drs. J. Morris and Y. Hayashi for valuable discussions, Dr. T. Hashimoto for DNA microarray analyses. This work was funded by Grants-in-Aid for Scientific Research (No. 23390040 to M. N and No. 25870634 to N. H). Disclosure of Summary: The authors have nothing to disclose.

\section{REFERENCES}

[1] Dietrich MO, Horvath TL (2013) Hypothalamic control of energy balance: insights into the role of synaptic plasticity. Trends Neurosci 36:65-73.

[2] Flak JN, et al. (2009) Chronic stress-induced neurotransmitter plasticity in the PVN. J Comp Neurol 517:156-65.

[3] Hatton GI (1997) Function-related plasticity in hypothalamus. Annu Rev Neurosci 20:375-97.

[4] Herman JP, Flak J, Jankord R (2008) Chronic stress plasticity in the hypothalamic paraventricular nucleus. Prog Brain Res 170:353-64.

[5] Horvath TL (2006) Synaptic plasticity in energy balance regulation. Obesity (Silver Spring) 14 Suppl 5:228s-233s.

[6] Keyser-Marcus L, et al. (2001) Alterations of medial preoptic area neurons following pregnancy and pregnancy-like steroidal treatment in the rat. Brain Res Bull 55:737-45.

[7] Celio MR, Blumcke I (1994) Perineuronal nets — a specialized form of extracellular matrix in the adult nervous system. Brain Res Brain Res Rev 19:128-45.

[8] Celio MR, et al. (1998) Perineuronal nets: past and present. Trends Neurosci 21:510-5.

[9] Pizzorusso T, et al. (2002) Reactivation of ocular dominance plasticity in the adult visual cortex. Science 298:1248-51.

[10] Gogolla N, et al. (2009) Perineuronal nets protect fear memories from erasure. Science 325:1258-61.

[11] Celio MR (1993) Perineuronal nets of extracellular matrix around parvalbumin-containing neurons of the hippocampus. Hippocampus 3:55-60.

[12] Hartig W, Brauer K, Bruckner G (1992) Wisteria floribunda agglutinin-labelled nets surround parvalbumin-containing neurons. Neuroreport 3:869-72.

[13] Nakamura M, et al. (2009) Expression of chondroitin sulfate proteoglycans in barrel field of mouse and rat somatosensory cortex. Brain Res 1252:117-29.

[14] Pantazopoulos H, et al. (2006) Subpopulations of neurons expressing parvalbumin in the human amygdala. J Comp Neurol 496:706-22.

[15] Seeger G, et al. (1996) Distribution patterns of Wisteria floribunda agglutinin binding sites and parvalbumin-immunoreactive 
neurons in the human visual cortex: a double-labelling study. J Hirnforsch 37: 351-66.

[16] Brauer K, et al. (1993) Distribution of parvalbumin-containing neurons and lectin-binding perineuronal nets in the rat basal forebrain. Brain Res 631:167-70.

[17] Bruckner G, et al. (1994) Cortical areas are revealed by distribution patterns of proteoglycan components and parvalbumin in the Mongolian gerbil and rat. Brain Res 658:67-86.

[18] Franklin KBJ, G Paxinos (2007) The Mouse Brain in Stereotaxic Coordinates. third ed. U.S.A: Academic Press. 360.

[19] Giamanco KA, Morawski M, Matthews RT (2010) Perineuronal net formation and structure in aggrecan knockout mice. Neuroscience 170: 1314-27.

[20] Carulli D, et al. (2010) Animals lacking link protein have attenuated perineuronal nets and persistent plasticity. Brain 133: 2331-47.

[21] Zhao C, Eisinger B, Gammie SC (2013) Characterization of GABAergic neurons in the mouse lateral septum: a double fluorescence in situ hybridization and immunohistochemical study using tyramide signal amplification. PLoS One 8:e73750.

[22] Szeidemann Z, Shanabrough M, Leranth C (1995) Hypothalamic Leu-enkephalin-immunoreactive fibers terminate on calbindin-containing somatospiny cells in the lateral septal area of the rat. J Comp Neurol 358: 573-83.

[23] Ding JM, et al. (1994) Proto-oncogene c-fos and the regulation of vasopressin gene expression during dehydration. Brain Res Mol Brain Res 21: 247-55.

[24] Sanchez E, et al. (2008) Differential effects of refeeding on melanocortin-responsive neurons in the hypothalamic paraventricular nucleus. Endocrinology 149:4329-35.

[25] Sharp FR, et al. (1991) c-fos mRNA, Fos, and Fos-related antigen induction by hypertonic saline and stress. J Neurosci $11: 2321-31$.

[26] Sheehan TP, Chambers RA, Russell DS (2004) Regulation of affect by the lateral septum: implications for neuropsychiatry. Brain Res Brain Res Rev 46:71-117.

[27] Anthony TE, et al. (2014) Control of stress-induced persistent anxiety by an extra-amygdala septohypothalamic circuit. Cell 156: $522-36$.

[28] Herman JP, et al. (2002) Role of the paraventricular nucleus microenvironment in stress integration. Eur J Neurosci 16:381-5.

[29] Poulain P (1983) Hypothalamic projection to the lateral septum in the guinea pig an HRP study. Brain Res Bull 10:309-13.

[30] Poulain P (1986) Properties of antidromically identified neurons in the enkephalinergic magnocellular dorsal nucleus of the guinea pig hypothalamus. Brain Res 362:74-82.

[31] Onteniente B, et al. (1989) Origin of the met-enkephalinergic innervation of the lateral septum in the rat. Cell Tissue Res 256 : 585-92. 